\title{
Low Gas Permeability of Particulate Films slows down the aging of gas marbles
}

\begin{abstract}
Yousra Timounay, ${ }^{a}$, Even Ou, ${ }^{a}$ Elise Lorenceau, ${ }^{a}$ and Florence Rouyer ${ }^{b}$
Including solid particles in liquid films drastically change their properties: "gas marbles" can resist overpressure and underpressure without deforming contrary to their pure liquid counterparts, also known as soap bubbles. Such gas marbles can therefore prove useful as gas containers able to support stresses. Yet, as their liquid counterparts, they can undergo gas transfer, which can reduce the scope of their applications. However, their permeability have never been characterized. In this paper, we measure the gas permeability of gas marbles through dedicated experiments. Our results show that particulate films are less permeable to gas than their pure liquid counterparts. We attribute this limited overall gas flux to the particles that reduce the surface area through which gas diffuses.
\end{abstract}

Liquid droplets or bubbles are promising tools to encapsulate small volumes of gas or liquid which can prove useful in various fields. For example, spray drying, where fine powders are produced by the rapid drying of aerosol droplet, has become the method of choice for manufacturing large quantities of pharmaceuticals ${ }^{1}$. In biology, aphids have developed an efficient way of handling their excreted honeydew droplet. By encapsulating them with a secreted powdery wax, they are able to convert the droplets into liquid marbles, that can be rapidly and efficiently moved $^{2}$. Such liquid marbles, which can be self-propelled by electric, magnetic or gradients of surface tension open a diversity of microfluidics applications ${ }^{3,4}$. In this spirit and inspired by the way nature encapsulates liquids, a start-up came up with an innovative way of packaging drinkable water by encircling water in an edible elastic membrane made of algae. These large drops have the convenience of plastic bottles while limiting the environmental impact (see http://oohowater.com/). In all these applications, bubbles/droplets must be stabilized under coalescence and meet specific features such as high mobility or sensitivity to an external field to induce controlled delivery ${ }^{5}$. As a common feature of submillimetric bubbles and drops is that they are governed by capillarity, hence an efficient way to modify their global properties is to tune their surface properties. To do so, additives of different sizes have been successfully incorporated to bubbles and drops interfaces. This has been proven useful to modify the mechanical properties of additive laden interfaces. At the molecular scale, macromolecules (such as proteins) or surfactants adsorbed at the interface delimiting a bubble/drop lower its intern overpressure (Laplace pressure) by lowering the surface tension, while droplets

\footnotetext{
a Université Paris-Est, Laboratoire Navier, UMR 8205 CNRS, ENPC ParisTech, IFSTTAR, 2 allée Kepler, 77420 Champs-Sur-Marne

${ }^{b}$ Université Paris-Est, Laboratoire Navier, UMR 8205 CNRS, ENPC ParisTech, IFSTTAR, 5 bd Descartes, 77454 Champs-Sur-Marne
}

coated by solid micrometric particles, known as liquid marbles, exhibits unusually high mobility ${ }^{6}$. The sensitivity to gas transfer processes is also modified in the presence of surface additives. At the molecular scale, the mass transfer of gas through foam films is modified by the presence of molecular surfactants. The common view is that when an additive is spread onto a quiescent liquid, an additional interfacial resistance to gas transfer arises from the adsorption of the surfactants onto the interfaces ${ }^{7,8}$. Therefore, the resistance to gas transfer of a single foam film constituted of an aqueous core sandwiched between two adsorbed monolayers of surfactant is the sum of the resistance of the monolayers and the resistance of the central liquid layer, which is proportional to the film thickness and the diffusion coefficient of the gas in the liquid phase ${ }^{9-11}$. At a nanoscale, adding nanoparticles or macromolecules at a fluid-fluid interface has been shown to induce surface elasticity that can stop coarsening following Gibbs criteria as highlighted at the scale of 2 bubbles ${ }^{12}$ or of the whole foam ${ }^{13}$. At a larger microscale, particulate bubbles can sustain non-spherical shapes ${ }^{14}$ or negative relative pressure in the order of Laplace pressure before collapsing. Their dissolution can even be stopped in degassed liquids ${ }^{15-18}$.

Despite these unusual properties, the permeability of liquid films decorated by solid particles has barely been investigated. This is mainly due to experimental difficulties. Indeed, most of the particle laden systems mentioned above are constituted of a single particle laden interface with inner and outer phases of different nature (a liquid inner phase and gaseous outer phase for drops or a gaseous inner phase and liquid outer phase for bubbles). Thus, particles are commonly only present at a single interface and such systems do not constitute liquid films. In this paper, we fill this gap by considering genuine particle laden bubbles also named gas marbles, where a gas volume is separated from the outer gaseous atmosphere by a particulate thin liquid 
film. Our particulate membrane consists in a monolayer of micrometric particles bridging the two interfaces of the thin liquid film. Such gas marbles have barely been investigated so far, yet, they exhibit interesting mechanical features due to the cohesion in the shell induced by the attractive interactions between the particles ${ }^{19}$. Consequently, such objects can sustain a significant overpressure before inflating and their collapse pressure is higher ( $\sim 10$ times greater) than the collapse pressure reported for bubbles/drops delimited by one interface. Moreover, at equilibrium, gas pressures inside and outside the gas marble are equal which is equivalent to a bubble with zero surface tension. Gas marbles are thus ideal candidates to transport gas as they are not subjected to coarsening since their inner pressure is not fixed by Laplace pressure. However, if the compositions of the inner and the outer gas are not the same or if a pressure difference between the inner and outer gas is imposed (out of equilibrium), the gas molecules may diffuse through the liquid phase of the thin particulate film, thus inducing the aging of the gas marble. In the present work, we study the diffusion of air through the particulate film delimiting a single gas marble and deduce its gas permeability. We show that particulate membranes are much less permeable to air than pure liquid films and we attribute this property to the particles that reduce the liquid/air interfaces area of films they are part of.

The liquid used to create gas marbles is an aqueous solution of Sodium Dodecyl Sulfate (SDS) at the critical micelle concentration $(0.2 \% \mathrm{w} / \mathrm{w})^{20}$. This concentration is chosen because it is a good compromise between the gas marble stability and the hydrophobicity of the particles. Indeed an increase of the SDS concentration would result in a more stable film but it would also decrease the hydrophobicity of the particles in contact with the solution and vice versa. The liquid's volumetric mass density is equal to 1000 and its surface tension is equal to 36 . The particles, in turn, are monosized polystyrene beads (Dynoseeds from Microbeads) with a volumetric mass density equal to 1050. Particles with two different diameters $D_{p}$ are used: 250 and 590. To modify their wetting properties, the particles have been immersed for 45 minutes into an octane solution containing dissolved silane $(1 \mathrm{H}, 1 \mathrm{H}, 2 \mathrm{H}, 2 \mathrm{H}$-Perfluorododecyltrichlorosilane, $97 \%$ from Sigma) at a concentration of $1 \mathrm{~g} / \mathrm{L}$. After the silanization, the contact angle between the particles and the SDS solution at the $\mathrm{CMC}$ is equal to $95^{\circ} \pm 10^{\circ}$ making them slightly hydrophobic. To create gas marbles, we immerse and withdraw a frame made of chrome wires of 60 diameter from a container filled with the SDS solution with a dense raft of particles on its interface. The frames we use are rectangular with a few millimeters length and width. The formation of the gas marble results from the destabilization of the particle laden interface initially driven by the frame. Due to its mass, the particle laden interface detaches from the frame and closes over itself creating a bubble. Gas marbles we generate using this technique are thus air bubbles in air environment and they are highly covered in particles (see figure 1-a and b).

To characterize the permeability of particulate films, we first increase the inner pressure of gas marbles delimited by those films by a few millibars. Then, we follow the spontaneous evolution of the inner pressure as a function of time. To do so, we use the set-up shown in figure 1-c, where the gas marble is connected to a syringe and to a pressure sensor. To increase the inner pressure of the gas marble quasi-statically, we decrease the volume of the syringe using a syringe pump. For all of our experiments, the flow rate $Q$ imposed by the syringe pump remained constant and equal to 0.06 . We also follow the shape of gas marbles during the experiments thanks to two cameras placed above and on the side of the gas marble.

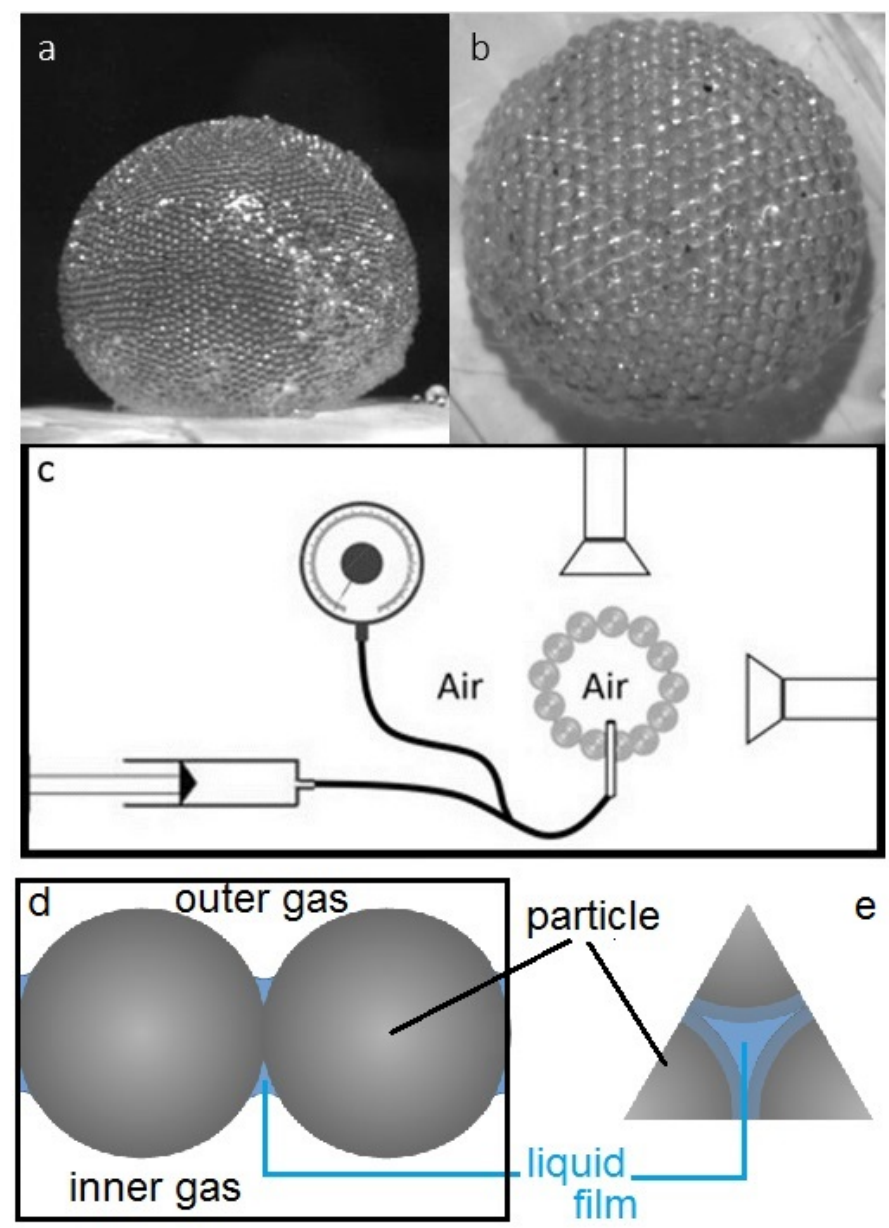

Fig. $1 \mathrm{a}$ and $\mathrm{b}$ are side and top views of a gas marble $\left(D_{p}=250\right)$ and $\mathrm{c}$ shows the experimental set up used to study the behavior of gas marbles in air during inflation or deflation. $d$ and e are sketches of the particle shell at the scale of the particles adapted from ${ }^{19}$.

Figure 2 shows an example of a plot of the pressure inside a gas marble relative to the atmospheric pressure $\Delta P=P-P_{a t m}$ as a function of time. $t=0$ corresponds to the moment when we stop decreasing the volume of the syringe connected to the gas marble. For $t<0$, the pressure inside the bubble increases linearly with time (cf. inset of Figure 2). Applying ideal gas law to the gas reservoir composed of the gas inside the syringe, the gas marble and the connecting tubes we can write the following: $\Delta P=P_{\text {atm }}\left[V_{i} /\left(V_{i}+Q \cdot t\right)-1\right]$ with $V_{i}$ is initial volume of the gas reservoir. Knowing that $V_{i} \sim 3$ and $Q \cdot t \sim 0.03$, ideal gas law can be simplified to the following linear expression matching thus the experimental result: $\Delta P \sim P_{\text {atm }} Q \cdot t / V_{i}$. As previously said, starting from $t=0$, the volume is kept constant ( $V=$ cst and $Q=0$ ) un- 


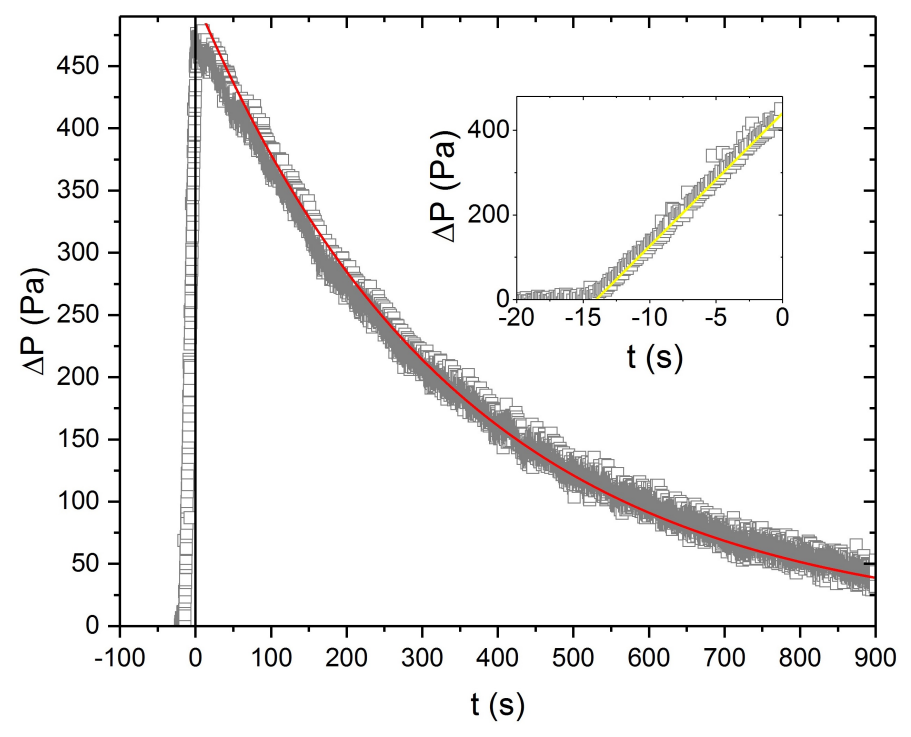

Fig. 2 Relative pressure inside a gas marble as a function of time with $D_{P}=250$. The red curve is as fit of equation 2 to the experimental data. The inset is a zoom on the relative pressure curve at the very first moments of the experiment during which the gas marble is pressurized by reducing the syringe's volume at a constant flow rate $Q$. The line corresponds to a linear approximation of the ideal gas law.

til the end of the experiment. Yet, we observe that the pressure inside gas marbles decreases spontaneously. Recalling that the volume is constant during the decrease of $\Delta P$ and knowing that our experimental set-up is tight, we impute the spontaneous evolution of the relative inner pressure of gas marbles to gas diffusion through the film delimiting them. The permeability of particulate films can be characterized by a coefficient $k$ defined as the following ${ }^{9}$ :

$$
\frac{\mathrm{d} C}{\mathrm{~d} t}=-\frac{k A}{V}\left(C-C_{a}\right)
$$

with $A$ the surface area of the particulate film through which the gas diffuses and $\Delta C=C-C_{a}$ the difference between the molar concentration of gas inside and outside the bubble.

Integrating equation 1 leads to: $C-C_{a}=\left(C_{0}-C_{a}\right) e^{-\frac{k A t}{V}}$ with $C_{0}$ the initial molar concentration inside the gas marble. Using the ideal gas law yields to the time-dependent equation for the relative pressure:

$$
\Delta P=\Delta P_{0} e^{-\frac{k A t}{V}}
$$

with $\Delta P_{0}$ the relative pressure inside the gas marble at $t=0$.

Using the method of least squares, we fit equation 2 to experimental pressure curves with the characteristic time $\tau=\frac{V}{k A}$ as an adjustable parameter. An example is presented in figure2. Repeating such experiments for different gas marbles and particle diameters (Figure3), we show that gas diffuses through the permeable particulate film that delimits the bubble and that relative pressure decreases with time following equation 2 . The measured characteristic times typically varies from 250 to 400 for slightly different gas marbles.

To go one step further, we determine the gas marble aspect

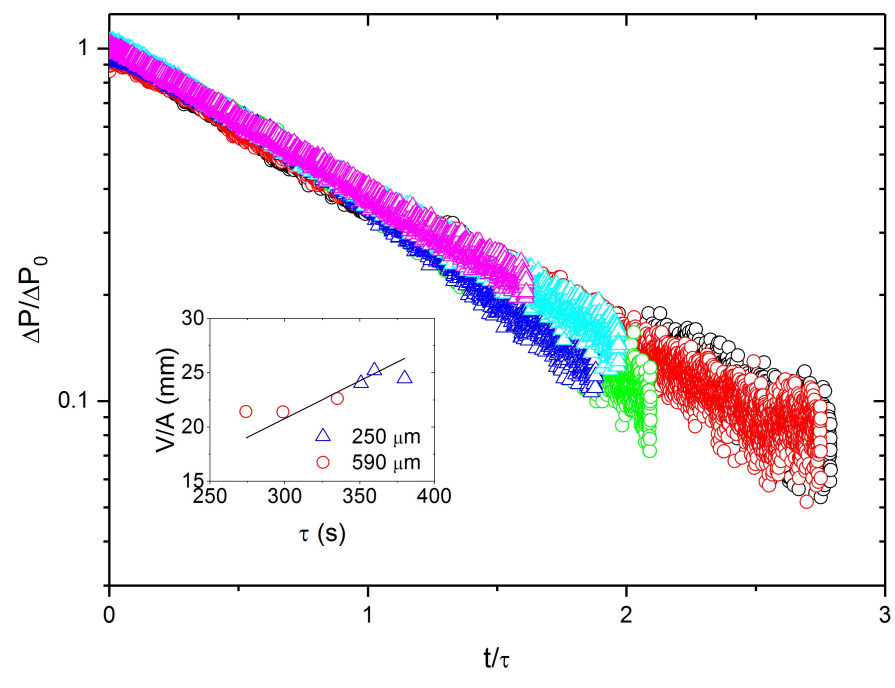

Fig. 3 Normalized relative pressure inside gas marbles as a function of time normalized by the characteristic time $\tau$. Circles correspond to gas marbles with 250 particles and triangles to 590 particles. Different colors indicate different experiments. The inset is a plot of $V / A$ as a function of $\tau$ and the line corresponds to a linear fit.

ratio $V / A$. In our experiments, air diffuses through the cap delimiting the bubble in contact with the atmosphere. This cap is not perfectly spherical as gas marbles deform slightly under their own weight. The exchange surface area $A$ is thus computed from the radius and the height of the spherical cap that we deduce from the images taken by the cameras. Note that the gas marbles we consider, do not deform during diffusion experiments. The height and the radius are thus constant for a given experiment. On another hand, we consider that $V \sim V_{i}$ with $V_{i}$ the sum of the volume of the gas marble, the volume of the syringe at $t=0$ and the volume of the connecting pipes. We deduce the numerical value of $V$ from the pressure dependance upon time during the inflation step (inset 2). In the inset of figure 3 , we plot $V / A$ as a function of the characteristic time $\tau$. Assuming a linear fit, we deduce the permeability of particle laden films $k=(0.069 \pm 0.009) \cdot 10^{-3}$.

The values of air permeability we deduce do not depend on the size of the particles and are ten times smaller than the permeability to air of a bare SDS soap film of equivalent thickness which is typically $\sim 10^{-39,11,21,22}$. The permeability value of bare soap films typically arises from the contribution of the two surfactants monolayers and the inner aqueous layer ${ }^{9}$. Adding solid particles in this sandwiched structure can have different consequences. First, it can modify the organization of this three-layer sandwich system due to the menisci that can appear at the contact line. Yet, in our system, the contact angle is around $90^{\circ}$ thus the liquid air interfaces are mainly flat as sketched in Fig. 1-d. Then, the values of $k$ we present are computed after assuming that air diffuses through the totality of the surface of the particulate film delimiting the marble in contact with the atmosphere. In reality, the diffusion area is much smaller. It corresponds to the area of the liquid film between the particles. Top and side pictures of gas marbles show that the particles are highly packed. They even reach the hexagonal packing as we can see in figure 
1-a and $\mathrm{b}$. The surface fraction of the particles on the films delimiting gas marbles is thus $\sim 0.91$. Considering this value of particles surface fraction and neglecting the additional surfaces induced by the menisci in between the particles, the exchange area through which air diffuses from inside gas marbles is the area of the thin liquid films (light blue in 1-e) and corresponds to $\sim 0.1 A$ rather than $A$. The new value of the permeability, noted $k_{f}=(0.69 \pm 0.09) \cdot 10^{-3}$ and thus qualitatively agree with the values of the permeability of soap films reported in the literature ${ }^{9}$. In this range of particle diameter and contact angle, the particles merely consist in reducing the exchange surface area.

We now discuss whether this ten-fold increase of the effective permeability can quantitatively explain the remarkable stability of aqueous foams stabilized by nanoparticles ${ }^{13,23-25}$. Cervantes et al. ${ }^{13}$ measured the temporal evolution of the average bubble radius of 3D foams stabilized by nanoparticles at different particles concentration from light transmission in multiple diffusion regime $^{13}$. They use Mullin's expression ${ }^{26}$ to fit the average radius of dry foam $R^{2}(t)=R_{0}^{2}\left(1+\left(t-t_{0}\right) / \tau_{c}\right)$, where $R_{0}$ is the value of the average bubble radius at $t_{0}$ and $\tau_{c}$ the characteristic time of coarsening. At relatively short time scale (i.e. for $0<t<10000$ s) and for aqueous foams stabilized by a dilute dispersion of silica nanoparticles at a concentration of $0.1 \%$ in weight, $\tau_{c} \sim 330 \mathrm{~s}$, while $\tau_{c} \sim 20000 \mathrm{~s}$ for foams stabilized by dispersion at $1 \%$ in weight. This $\tau_{c} \sim 20000 \mathrm{~s}$ value, determined from the fit of the data provided by Cervantes et al. is 50 times smaller than the value provided in the original article due to the limited time-scale $t<10000$ s we considered. Indeed, at larger time scales, the liquid fraction of the particle laden foam turns out to be extremely small due to gravity drainage thus increasing the concentration of particles at the interface. In this regime, coarsening is totally inhibited by surface elasticity of the particle laden interfaces as shown in ${ }^{13}$. To understand whether these $330 \mathrm{~s}$ and 20000s coarsening time scale values can be explained by the difference in surface particle coverage, we recall that in Mullin's expression, $\tau_{c}$ is inversely proportional to the film permeability, and proportional to the film thickness and a structural foam correction factor which depends on the initial bubble radius and the liquid fraction of the foam. Thus, the observed 20000/330 $=60$-fold increase in coarsening timescale can be partially explained by our findings as a 10 -fold decrease in the exchange area between the two foams induces a decrease of 10 on the effective film permeability.

To conclude, gas marbles have a low gas permeability due to the reduction of liquid film area by the particle coverage because gas exchange occurs through the liquid phase. In the present case of high particle packing of the shell, gas permeability is reduced by a factor of 10 . Such results would explain the slow down of coarsening for foam stabilized by particles, before coarsening is stopped at very large particle concentration due to surface elasticity of the interfaces.

Acknowledgment We thank Olivier Pitois and Emmanuelle Rio for fruitful discussions. Y.T also acknowledges financial support form IFSTTAR.

\section{References}

1 C. Bosquillon, C. Lombry, V. Preat and R. Vanbever, J. Controlled Release, 2001, 70, 329-339.

2 N. Pike, D. Richard, W. Foster and L. Mahadevan, Proc. Roy. Soc. B, 2002, 269, 1211-1215.

3 P. Aussillous and D. Quere, Proc. Roy. Soc. A, 2006, 462, $973-$ 999.

4 E. Bormashenko, Langmuir, 2017, 33, 663-669.

5 J. Eow, M. Ghadiri, A. Sharif and T. Williams, Chem. Eng. Journal, 2001, 84, 173-192.

6 P. Aussillous and D. Quéré, Nature, 2001, 411, 924-927.

7 I. Langmuir and D. B. Langmuir, J.Phys. Chem., 1927, 31, 1719.

8 J. Hawke and A. a. Alexander, Retardation of evaporation by monolayers: transport process., New-Tork Academic Press, New-York, 1962.

9 H. Princen and S. Mason, Journal of Colloid Science, 1965, 20, 353-375.

10 H. Princen, J. Overbeek and S. Mason, Journal of Colloid Science, 1967, 24, 125-130.

11 R. Farajzadeh, R. Krastev and P. L. J. Zitha, Advances in colloid and interface science, 2008, 137, 27-44.

12 A. Salonen, C. Gay, A. Maestro, W. Drenckhan and E. Rio, EPL (Europhysics Letters), 2016, 116, 46005.

13 C. Martinez, A., E. Rio, G. Delon, A. Saint-Jalmes, D. Langevin and P. Binks, Bernard, Soft Matter, 2008, 4, 1531-1535.

14 A. B. Subramaniam, M. Abkarian, L. Mahadevan and H. A. Stone, Nature, 2005, 438, 930-930.

15 M. Abkarian, A. B. Subramaniam, S.-H. Kim, R. J. Larsen, S.M. Yang and H. A. Stone, Physical Review Letters, 2007, 99, 188301.

16 O. Pitois, M. Buisson and X. Chateau, The European Physical Journal E, 2015, 38, 1-7.

17 N. Taccoen, F. Lequeux, D. Z. Gunes and C. N. Baroud, Physical Review X, 2016, 6, 011010.

18 C. Monteux, J. Kirkwood, H. Xu, E. Jung and G. G. Fuller, Physical Chemistry Chemical Physics, 2007, 9, 6344-6350.

19 Y. Timounay, O. Pitois and F. Rouyer, Physical Review Letters, 2017, 118, 329-339.

20 P. Mukerjee and K. J. Mysels, Critical micelle concentrations of aqueous surfactant systems, Dtic document technical report, 1971.

21 M. Ramanathan, H.-J. Mueller, H. Moehwald and R. Krastev, ACS Applied Materials \& Interfaces, 2011, 3, 633-637.

22 R. Krustev, D. Platikanov and M. Nedyalkov, , 1997, 123, 383390.

23 T. E. Gonzenbach U. T., Studart A. R. and G. L. J., Angew.Chem.Int.Ed, 2006, 45, 3526-3530.

24 B. P. Binks and T. S. Horozov, Angew.Chem.Int.Ed, 2005, 44, 3722-3725.

25 A. Stocco, E. Rio, B. P. Binks and D. Langevin, Soft Matter, 2011, 7, 1260-1267.

26 W. Mullins, J.Applied Phys., 1986, 59, 1341-1349. 